\title{
Conflito no desempenho do papel de mãe em estudos com mães de recém-nascidos hospitalizados: revisão integrativa
}

\author{
MOTHER ROLE CONFLICTS IN STUDIES WITH MOTHERS OF HOSPITALIZED \\ NEWBORNS: AN INTEGRATIVE REVIEW
}

\section{CONFLICTO EN EL DESEMPEÑO DEL PAPEL MATERNAL EN ESTUDIOS CON MADRES DE RECIÉN NACIDOS HOSPITALIZADOS: REVISIÓN INTEGRAL}

\section{Elenice Valentim Carmona ${ }^{1}$, Kelly Pereira $\mathrm{Coca}^{2}$, lanê Nogueira do Vale ${ }^{3}$, Ana Cristina Freitas de Vilhena Abrão ${ }^{4}$}

\section{RESUMO}

A hospitalização de um recém-nascido provoca a separação entre mãe e filho quando o contato é fundamental para o desenvolvimento do papel materno. Assim, as mães tendem a se sentir incapazes de atender as necessidades dos filhos, enfrentando dificuldades para lidar com seus sentimentos e com as demandas dos familiares. $O$ objetivo deste estudo foi identificar as Características Definidoras do Diagnóstico de Enfermagem Conflito no desempenho do papel de mãe em estudos sobre a experiência de ser mãe na unidade neonatal. Trata-se de uma revisão integrativa da literatura, que utilizou 15 estudos qualitativos, publicados de 2004 a 2009 em periódicos indexados na Literatura Latino-Americana e do Caribe em Ciências da Saúde e Medical LiteratureandRetrieval System OnLine. Este diagnóstico é um fenômeno de enfermagem a ser estudado por enfermeiros neonatais para que possam reconhecer e propor intervenções às necessidades maternas, visto que nove das dez Características Definidoras foram identificadas nos depoimentos das mães.

\section{DESCRITORES}

Relações mãe-filho

Recém-nascido

Hospitalização

Criança hospitalizada

Comportamento materno

Diagnóstico de enfermagem

\begin{abstract}
The hospitalization of a newborn separates mother and child at a time when their contact is essential for developing the mother's role. Therefore, mothers tend to feel incapable of meeting their child's needs, and face difficulties in dealing with their personal feelings and the demands of their family. The objective of this study was to identify the defining characteristics of the nursing diagnosis Conflict in performing the role of mother in studies addressing the experience of being a mother in the neonatal unit. This is an integrative literature review, which utilized 15 qualitative studies published between 2004 and 2009, in journals indexed in the Latin American and Caribbean Health Sciences Literature and on the Medical Literature and Retrieval System Online. This diagnosis is a nursing phenomenon to be studied by neonatal nurses so they are able to recognize and propose interventions to meet the mothers' needs, considering that nine out of ten defining characteristics were identified in the mothers' statements.
\end{abstract}

\author{
DESCRIPTORS \\ Mother-child relations \\ Infant, newborn \\ Hospitalization \\ Child, hospitalized \\ Maternal behavior \\ Nursing diagnosis
}

\section{RESUMEN}

La hospitalización del neonato provoca separación entre madre e hijo cuando el contacto es fundamental para el desarrollo del papel materno. Consecuentemente, las madres tienden a sentirse incapaces de atender las necesidades del hijo, tienen dificultad para afrontar el sentimiento y las demandas familiares. Se objetivó identificar Características Definidoras del Diagnóstico de Enfermería Conflicto en el desempeño del papel maternal en estudios acerca de la experiencia de ser madre en la unidad neonatal. Revisión integral de la literatura, habiéndose utilizado quince estudios cualitativos, publicados entre 2004-2009 en períodos indexados en la Literatura Latinoamericana y del Caribe en Ciencias de Salud y Medical Literature and Retrieval System Online. Este diagnóstico constituye un fenómeno de Enfermería a ser estudiado por enfermeros neonatales para que puedan reconocer y proponer intervenciones para las necesidades maternas, en virtud de que nueve de las diez Características Definidoras fueron identificadas en los testimonios de las madres

\author{
DESCRIPTORES \\ Relaciones madre-hijo \\ Recién nacido \\ Hospitalización \\ Niño hospitalizado \\ Conducta materna \\ Diagnóstico de enfermería
}

${ }^{1}$ Enfermeira. Doutoranda em Enfermagem pela Universidade Federal de São Paulo. Professora do Departamento de Enfermagem da Faculdade de Ciências Médicas da Universidade Estadual de Campinas. Campinas, SP, Brasil. elenice@fcm.unicamp.br ${ }^{2}$ Enfermeira. Doutoranda em Enfermagem pela Universidade Federal de São Paulo. Coordenadora do Curso de Especialização em Enfermagem Obstétrica do Centro Universitário São Camilo. São Paulo, SP, Brasil. kcocaepm@hotmail.com ${ }^{3}$ Enfermeira. Professora Doutora do Departamento de Enfermagem da Faculdade de Ciências Médicas da Universidade Estadual de Campinas. Campinas, SP, Brasil. ianenvale@gmail.com. ${ }^{4}$ Professora Doutora do Departamento de Enfermagem da Universidade Federal de São Paulo. São Paulo, SP, Brasil. ana.abrao@unifesp.br 


\section{INTRODUÇÃO}

A prematuridade (nascimento com idade gestacional menor que 37 semanas) e o baixo peso ao nascer (peso menor que $2500 \mathrm{~g}$ ) são as causas mais frequentes de hospitalização de bebês em países em desenvolvimento, como no Brasil, o que está associado a maior risco de complicações e óbito neonatal. Estas causas são desencadeadas por más condições sociais e econômicas, infecções e um deficiente atendimento pré-natal ${ }^{(1)}$.

Com os avanços da assistência à saúde, tem diminuído a mortalidade dos bebês prematuros e enfermos, aumentando o número de crianças com doenças graves e crônicas. Os RNs (recém-nascidos) são pacientes de maior fragilidade clínica, principalmente os prematuros e de baixo peso, e passam por longos períodos de hospitalização ${ }^{(2-4)}$. No contexto da assistência neonatal, assim como os pequenos pacientes batalham por suas vidas, as mães vivenciam a hospitalização como uma situação difícil e, muitas vezes, inesperada. Tendem a se perceberem como incapazes para atender as necessidades do filho, apresentam dificuldades para lidar com seus sentimentos e precisam ainda lidar com demandas de outros familiares ${ }^{(2)}$.

Ao longo de consultas à literatura, verificou-se que muitos autores, que estudam a experiência de mulheres que têm seus filhos recém-nascidos hospitalizados, descrevem relatos maternos que apresentam Características Definidoras (CDs) do Diagnóstico de Enfermagem (DE) Conflito no desempenho do papel de mãe, sem nomeá-las como tal. Tal observação desencadeou o interesse em desenvolver investigação específica sobre a presença destas CDs nos estudos qualitativos que abordam tal temática.

O diagnóstico em questão é definido, segundo publicação da NANDA-| ${ }^{(5)}$ (North American Nursing Diagnosis Association), atualmente denominada NANDA Internacional), versão 2009-2011, como: mãe experimenta confusão e conflito no desempenho de seu papel em resposta a uma crise. Este DE apresenta as seguintes CDs (sinais ou sintomas observáveis ou comunicáveis que representam a presença do diagnóstico):

\footnotetext{
Ansiedade; Distúrbio demonstrado nas rotinas de cuidado; Mãe expressa preocupação a respeito da perda percebida de controle sobre decisões relacionadas ao filho; Medo; Mãe expressa preocupação(ões) em relação à família (p.ex., funcionamento, comunicação, saúde); Mãe expressa preocupação(ões) em relação a mudanças no papel materno; Mãe expressa sentimento(s) de inadequação para atender às necessidades do filho (p.ex. físicas e emocionais); Relutante em participar de atividades usuais de cuidado, mesmo com encorajamento e apoio; Mãe verbaliza sentimentos de culpa e Mãe verbaliza sentimentos de frustração.
}

DE é um julgamento clínico sobre respostas do indivíduo, família ou comunidade a problemas de saúde/processos vitais reais ou potenciais. Ele proporciona a base para seleção de intervenções de enfermagem que alcancem resultados satisfatórios para o paciente, pelos quais o enfermeiro é responsável. Trata-se de um processo cognitivo através do qual se fazem inferências quanto a dados obtidos sobre o paciente, focalizando as respostas apresentadas por ele $\mathrm{e}^{(5)}$. O uso de $\mathrm{DE}$ relacionados à mãe na unidade de internação neonatal ainda pode ser considerado incipiente, uma vez que os enfermeiros neonatais são muito voltados para os diagnósticos médicos e para a assistência ao RN.

A construção do papel materno é um processo cognitivo-afetivo complexo, que tem suas origens na própria experiência da mãe enquanto filha e gradualmente é processado ao longo da gestação. E que continua sendo construído e modificado no contexto de interações constantes com o bebê, pelo qual a mulher tem total responsabilidade. Nestas interações o bebê desempenha um importante papel, visto que regula os comportamentos maternos a partir de suas respostas ${ }^{(6-7)}$. E o desenvolvimento do apego é uma experiência profunda e complexa que requer contato físico precoce ${ }^{(6,8)}$. Em contraste a isto, as mães de RN hospitalizados têm contatos mais esporádicos e limitados com o filho, sendo que o desenvolvimento deste relacionamento pode ser adiado. Além disso, em função da gravidade do estado clínico do bebê, o apego pode ser realmente comprometido como mecanismo de defesa materno(8-10).

Ao longo do processo de assumir o cuidado do filho hospitalizado, as mães costumam relatar sentimentos de baixa autoestima, insegurança, fracasso e inabilidade para superar as dificuldades que surgem da experiência de cuidar. Elas referem dúvidas quanto à própria competência para cuidar de seus fiIhos, reconhecer e resolver problemas ${ }^{(11)}$. Enfrentam ainda desafios adicionais que interferem no funcionamento da família como: o significado da hospitalização do bebê; a separação entre os membros da família; outros filhos para cuidar; problemas de saúde; necessidade de grande suporte psicológico para a família e aumento de preocupações financeiras ${ }^{(8-10)}$.

Neste contexto, considera-se incontestável que o cuidado aos RN deve abranger o cuidado às mães. Logo, também devem ser identificados DE maternos em unidades neonatais, uma vez que possibilitarão oferecer assistência com o objetivo de dar melhores condições para as próprias mães assumirem os cuidados de seus filhos. Estando elas também assistidas, poderão contribuir mais para o restabelecimento dos bebês e assumir plenamente os cuidados futuros.
Conflito no desempenho do papel de mãe em estudos com mães de recém-nascidos hospitalizados: revisão integrativa Carmona EV, Coca KP, Vale IN, Abrão ACFV 
Para se planejar a assistência de enfermagem é importante reconhecer o fenômeno que requer atenção. Considera-se que chamar a atenção para este DE tem grande valor neste contexto assistencial, porque, se a resposta humana não for identificada e não receber intervenções, a mãe pode desenvolver Maternidade prejudicada ${ }^{(12)}$ : diagnóstico que se descreve situação na qual existe incapacidade do cuidador primário de criar, manter ou reaver um ambiente que promova ótimo crescimento e desenvolvimento da criança $^{(5)}$. Além disso, embora não seja possível afirmar a presença do Conflito no desempenho do papel de mãe nos estudos analisados, a importância deste trabalho baseia-se em demonstrar como dados subjetivos, advindos de estudos com abordagem qualitativa, podem subsidiar o estudo de fenômenos de Enfermagem, auxiliando no desenvolvimento da linguagem padronizada para melhor descrição destes, de forma a torná-los cada vez mais úteis à prática clínica. A unidade neonatal ainda se mostra carente de estudos desta natureza.

Este estudo teve como objetivo analisar, por meio de revisão integrativa da literatura, as Características Definidoras do Diagnóstico de Enfermagem Conflito no desempenho do papel de mãe nos relatos de mães, sujeitos de estudos qualitativos, sobre a experiência de ser mãe na unidade de internação neonatal.

\section{MÉTODO}

Trata-se de uma revisão integrativa da literatura ${ }^{(13)}$ com trabalhos que abordam a experiência da mulher enquanto mãe de recém-nascido hospitalizado. A revisão integrativa é um método de análise de pesquisas relevantes que dão suporte para a tomada de decisão e melhoria da prática clínica. De acordo com este método, a primeira etapa foi a identificação do tema e elaboração de uma hipótese a ser testada: verificar se estudos que abordam a experiência de ser mãe no contexto da unidade de internação neonatal apresentavam CDs do DE Conflito no desempenho do papel de mãe em seus resultados.

A etapa seguinte foi o estabelecimento de critérios para inclusão e exclusão de estudos, bem como sua busca na literatura. Para tanto, foram utilizadas as seguintes fontes de referência para a seleção dos estudos: Literatura LatinoAmericana e do Caribe em Ciências da Saúde (LILACS) e Medical Literature and Retrieval System On Line (MEDLINE).

Os descritores utilizados para busca foram: Relações mãe-filho; Comportamento materno; Recém-nascido; Hospitalização; Criança hospitalizada; Pesquisa qualitativa e respectivas versões em Inglês. Tais descritores foram definidos a partir de consulta aos Descritores em Ciências da Saúde da Biblioteca Virtual e Saúde. Palavras que não são descritores exatos também foram utilizadas: mãe acompanhante; maternagem; recém-nascido hospitalizado, mãe e papel materno.
Foram considerados os seguintes critérios de inclusão para os estudos:

- Estudos publicados de 2004 a fevereiro de 2009, em periódico indexado nas bases de dados LILACS e MEDLINE, escritos em Inglês ou Português;

- Abordagem metodológica qualitativa, com uso de entrevista para coleta de dados;

- Foco na experiência da mãe na unidade de internação neonatal, sendo a mãe o sujeito de pesquisa e, portanto, submetida à entrevista;

- Presença de pelo menos um enfermeiro como coautor do trabalho.

Foram considerados como critérios de exclusão: estudos em que as entrevistas ocorreram após a alta do filho da unidade neonatal; artigos de revisão; trabalhos que abordavam a experiência materna no lar, após a hospitalização; trabalhos sem resumo disponível; trabalhos não disponíveis na íntegra e trabalhos em que a amostra foi submetida ao Método Canguru, por considerar-se uma vivência bastante diferente em relação às realidades em que não há possibilidade de tal contato. Outro critério de exclusão, estabelecido ao longo da coleta de dados, foi o fato de o estudo não ter o método claramente descrito.

Na revisão integrativa da literatura é importante definir as informações a serem extraídas dos estudos selecionados. Portanto, foi desenvolvido um instrumento de coleta de dados, para ser preenchido para cada artigo selecionado, contemplando os seguintes dados: referência bibliográfica; base de dados de indexação; objetivo e método. Além disso, o instrumento continha as CDs do diagnóstico em questão para serem assinaladas com $x$ em caso de estarem presentes nos resultados do trabalho. $O$ instrumento continha campos para registro de observações.

Realizou-se o levantamento bibliográfico para obtenção das referências das pesquisas, utilizando os descritores e palavras mencionados. Os resumos obtidos nas bases de dados foram lidos, buscando-se identificar artigos compatíveis com o objetivo. Então, ocorreu a leitura dos artigos selecionados, bem como preenchimento do instrumento para cada um deles. Os instrumentos preenchidos foram revistos quanto à legibilidade e coerência das informações. Os dados forão extraídos dos resultados e discussão dos estudos.

Cada artigo foi lido por pelo menos duas autoras, que realizaram o preenchimento do instrumento separadamente, ou seja, dois instrumentos eram preenchidos para cada artigo. E, no caso de discordância entre elas quanto à presença ou ausência de uma determinada CD, ocorria a leitura e o preenchimento do instrumento por uma terceira autora para chegar-se a um consenso. 


\section{RESULTADOS}

Utilizando-se os descritores e palavras-chave mencionados foram encontradas 124 referências: 85 no MEDLINE e 39 no LILACS. A partir da leitura dos resumos, foram selecionados 37 artigos para a leitura na íntegra: 17 do total de resumos pesquisados no MEDLINE e 20 do LILACS.

Após leitura dos 37 artigos, 15 trabalhos puderam realmente fazer parte do estudo. Isto porque somente ao longo de tal leitura foi possível verificar que muitos não atendiam aos critérios de inclusão, uma vez que detalhes do método estiveram melhor descritos no artigo que no resumo.

Os estudos objetivaram, em geral, investigar a vivência das mães de bebês hospitalizados em unidade neonatal, seus sentimentos e a experiência da maternidade em tal contexto. Os dados foram coletados por meio de entrevistas semi-estruturadas, que foram gravadas. Tiveram amostras de 6 a 26 mulheres. Quanto à análise dos dados, oito estudos realizaram-na segundo a Análise de Conteúdo de Bardin, três utilizaram a Fenomenologia, dois a Teoria Fundamentada nos Dados, um a Teoria do Vínculo de Pichon-Riviére e um deles a Etnografia. Dez estudos foram desenvolvidos por enfermeiros brasileiros e cinco por enfermeiros de outras nacionalidades.

O DE Conflito no desempenho do papel de mãe tem $10 \mathrm{CDs}$, segundo a Classificação atual da NANDA-I(5). É interessante salientar que apenas uma delas (Distúrbio demonstrado nas rotinas de cuidado) não esteve presente nas falas das entrevistadas, enquanto nove apresentaram frequência relevante nos depoimentos maternos (Tabela 1).

Tabela 1 - Características Definidoras do DE Conflito no desempenho do papel de mãe e sua frequência nos 15 artigos selecionados - São Paulo, 2009

\begin{tabular}{l|c}
\multicolumn{1}{c|}{ Características definidoras } & N=15 \\
\hline \begin{tabular}{l}
\multicolumn{1}{c}{ Medo } \\
Mãe expressa preocupação(ões) em relação a mudan- \\
ças no papel materno
\end{tabular} & 14 \\
$\begin{array}{l}\text { Mãe verbaliza sentimentos de culpa } \\
\text { Mãe expressa sentimento(s) de inadequação para aten- }\end{array}$ & 8 \\
$\begin{array}{l}\text { der às necessidades do filho } \\
\text { Mãe expressa preocupação(ões) em relação à família } \\
\text { Mãe verbaliza sentimentos de frustração }\end{array}$ & 6 \\
$\begin{array}{l}\text { Ansiedade } \\
\text { Relutante em participar de atividades usuais de cuida- } \\
\text { do, mesmo com encorajamento e apoio }\end{array}$ & 6 \\
$\begin{array}{l}\text { Mãe expressa preocupação a respeito da perda percebi- } \\
\text { da de controle sobre decisões relacionadas ao filho }\end{array}$ & 3 \\
\hline
\end{tabular}

\section{DISCUSSÃO}

O confronto entre o DE em questão e os dados da literatura demonstra a pertinência de seu conteúdo para descrever a situação da mulher que vivencia a hospitalização de um filho recém-nascido, visto que se propõe a descrever o conflito que ela experimenta para desempenhar seu papel junto ao filho, enquanto também passa por momento de crise. As CDs, sem dúvida, trazem sinais importantes de que a mulher precisa receber suporte para sentir-se mais confiante e melhor desempenhar seu papel de mãe. Este estudo não teve o objetivo de discutir as intervenções para o fenômeno, mas de evidenciar a presença destes sinais nos relatos maternos.

A seguir as CDs são discutidas em ordem decrescente de frequência na amostra de estudos, conforme apresentado na tabela acima.

\section{Medo}

Esta CD foi a mais presente nas falas das mulheres. 0 medo mostrou-se relacionado a temores sobre o futuro do bebê, especificamente a sequelas e sobrevivência. Foi o sentimento predominante para as mães e esteve relacionado sobretudo à morte do filho. Elas se referiram também a outros fatores que desencadeavam medo como os equipamentos e dispositivos utilizados no bebê, o ambiente da unidade neonatal, assim como a aparência e a fragilidade do filho(11,14-26).

\section{Mãe expressa preocupação(ões) em relação a mudanças no papel materno}

As mulheres relatam não se sentirem completamente mães por não serem responsáveis pelo cuidado do filho, por não poderem cuidar à sua maneira e ter com o filho a relação que imaginaram. Descrevem o distanciamento em relação ao filho, devido à internação, como uma experiência absolutamente diferente da idealizada por elas. Além disso, falam sobre o desconforto gerado pela percepção de que não são importantes e necessárias para o próprio filho $^{(11,15-16,18,21-27)}$.

As entrevistadas falam da dificuldade de desempenhar o papel de mãe devido a não saber como cuidar do próprio filho. Alguns depoimentos trazem um sentir-se inválida como mãe $e^{(22)}$, enquanto em outras falas esta experiência é descrita como não estar sendo mãe(18). Declaram terem ouvido do marido que não serviam nem para produzir leite, o que reforçou a percepção de que seu papel materno não estava sendo exercido(18).

As falas maternas descrevem o quanto a mudança no papel materno está relacionada a não terem visto o fiIho nascendo e não terem vivenciado todas as sensações do parto e do cuidado ao filho. As mulheres questionam como podem se sentir mães em tais condições de afastamento e sentimento de inutilidade. Outras comentam
Conflito no desempenho do papel de mãe em estudos com mães de recém-nascidos hospitalizados: revisão integrativa Carmona EV, Coca KP, Vale IN, Abrão ACFV 
que sentiram que o início do processo de apropriação do papel de mãe foi bastante postergado ${ }^{(15)}$. Uma mãe menciona que, devido a este distanciamento e mudanças no papel de mãe que esperava desempenhar, passou a realmente evitar cuidar do filho e ter contato com ele, temendo o apego $^{(24)}$.

\section{Mãe verbaliza sentimentos de culpa}

O sentimento de culpa esteve relacionado pelas mães a algo que fizeram ou que deixaram de fazer (por exemplo, o acompanhamento pré-natal) que pode ter prejudicado seus filhos, desencadeando um problema de saúde e a necessidade de internação logo após o nascimento $^{(14,16,18,20,22-25)}$.

Esta culpa se mostrou vinculada por algumas mães também ao recebimento de um castigo por algo que fizeram de muito errado a outrem, pecado este descrito como muito crítico para merecer tal punição. A punição é a hospitalização do filho recém-nascido e o consequente sofrimento para mãe, filho e família(20,22).

A culpa também esteve relacionada ao desejo de deixar o hospital e ir para casa ficar com os outros familiares, seja pela necessidade emocional da mulher de estar junto a eles, seja pela necessidade de oferecer-lhes cuidado. A culpa foi decorrente também da percepção de que não atendiam adequadamente tanto as necessidades do filho hospitalizado quanto as dos familiares ao longo deste processo $^{(25)}$. Este sentimento foi relacionado também a não terem sentido um vínculo imediato com o filho, como esperavam sentir ${ }^{(18)}$.

\section{Mãe expressa sentimento(s) de inadequação para aten- der as necessidades do filho}

As mães falam sobre uma falta de confiança na própria capacidade de cuidar de um bebê com necessidades especiais; algumas se referindo a uma dificuldade emocional, enquanto outras a problemas financeiros, falta de habilidade e de destreza para desempenhar os cuidados diretos ao filho(11,16,18,21-22,25-26).

\section{Mãe expressa preocupação(ões) em relação à família}

Como descrito nos exemplos entre parênteses que acompanham esta CD na Classificação da NANDA-I(5), os discursos maternos apresentam preocupações relativas ao funcionamento da família na sua ausência e em decorrência da hospitalização do filho, preocupam-se com a dinâmica do dia-a-dia, com questões financeiras, com a comunicação entre os familiares, assim como com a saúde de algum familiar em especial. A preocupação predominante é com o cuidado de outros filhos ${ }^{(15,21-23,25-26)}$.

As mães falaram sobre dificuldades para explicar a condição clínica do filho para os demais familiares ${ }^{(22)}$. Algumas mães declaram que familiares Ihe cobravam, além de informação, resultados satisfatórios, o que gerou angústia e preocupação adicionais ${ }^{(22)}$.

Os depoimentos maternos demonstram o quanto a mãe se sente em dívida para com sua família, sobretudo com outros filhos, ao mesmo tempo em que sente que não passa tempo suficiente com o filho hospitalizado. O conflito de papéis e responsabilidades faz com que elas considerem difícil estar o tempo necessário com o filho hospitalizado(21,26). As mães discorrem ainda sobre o impacto que a hospitalização do RN teve sobre a família e o conflito que sentem por desejarem estar com o RN e ao mesmo tempo com os outros familiares, principalmente outros filhos. Algumas descreveram um verdadeiro e intenso sentimento de solidão ${ }^{(25)}$. Todas estas preocupações também se relacionam ao sentimento de culpa mencionado tantas vezes por elas.

\section{Mãe verbaliza sentimentos de frustração}

O sentimento de frustração descrito pelas muIheres foi relacionado ao fato de todo o processo de nascimento e a hospitalização ser absolutamente diferente do que idealizaram e desejaram ${ }^{(14,19,22-23,25)}$. Ficar distante do filho logo após o nascimento foi algo descrito como muito frustrante ${ }^{(25)}$. O nascimento do filho em tais condições foi mencionado como um sonho não realizado, pois esperavam um nascimento normal(19).

\section{Ansiedade}

As mulheres descrevem um sentimento incômodo desencadeado por várias questões e o nomeiam como ansiedade. Esta aparece relacionada ao desejo de ir para casa, de ver os primeiros resultados positivos do tratamento do filho e de que o filho tenha alta hospitalar em breve. Este sentimento também foi descrito pelas mulheres como relacionado à percepção de que a produção de leite estava diminuindo ${ }^{(14-15,21,24-25)}$.

A palavra ansiedade apareceu muitas vezes na fala dessas mães para descrever a situação e os sentimentos vivenciados por elas no processo de maternagem de um filho hospitalizado. A NANDA-I(5) apresenta um DE intitulado Ansiedade que é definido como um vago e incômodo sentimento de desconforto ou temor, acompanhado por resposta autonômica. A fonte é frequentemente não específica ou desconhecida para o indivíduo. Trata-se de um sentimento de apreensão causada pela antecipação de perigo, um sinal de alerta que chama a atenção para um perigo iminente e permite ao indivíduo tomar medidas para lidar com a ameaça.

Os discursos destas mulheres não se referiram a respostas autonômicas, que muito bem caracterizam a ansiedade, mas sem dúvida elas vivenciaram os desconfortos e temores causados pela antecipação de um perigo ao qual teriam que reagir. Esta $C D$, em especial, precisa 
de investigação bastante cuidadosa para poder ser apontada na prática clínica.

\section{Relutante em participar de atividades usuais de cuida- do, mesmo com encorajamento e apoio}

As mães declararam que só estarão aptas a cuidar do filho após a alta hospitalar, demonstrando a falta de percepção sobre o processo necessário para tal. Comparam sua habilidade com a dos profissionais de enfermagem e, assim, afirmam ser complicado cuidar como os profissionais cuidam. Aqui parecem acreditar que existe uma forma específica de cuidar do filho, não se sentindo aptas para tal $\left.\right|^{(15,18,24)}$.

Seus depoimentos demonstram que estão comprometidas com o cuidado, mas se sentem incapazes de realizá-lo devido à situação em que se encontra o RN e ao próprio despreparo para participar do cuidado. Um dos estudos $^{(15)}$ apresentou uma fala materna que expressou bem este dado: O problema é comigo, 'né'? Eu tenho muita insegurança, mas eles me incentivam bastante!

\section{Mãe expressa preocupação sobre a perda percebida de controle sobre decisões relacionadas ao filho}

As mães disseram que sentem como se a equipe de enfermagem tivesse a custódia do bebê e, assim, estivesse sempre no controle. As mães lamentam o fato de precisarem pedir permissão até para tocar o próprio filho ${ }^{(11,18,25-26)}$.

Interessante que esta fala não apareceu nos estudos com mães brasileiras, o que pode ser explicado pelas diferenças culturais, pelas mães de outros países terem mais clareza sobre seus direitos no acompanhamento de um bebê hospitalizado, incomodando-se mais, portanto, com as regras e limitações impostas pela equipe de saúde.

\section{Distúrbio demonstrado nas rotinas de cuidado}

Esta CD não esteve presente nas falas maternas dos estudos incluídos, o que provavelmente esteja relacionado a se tratar de um evento a ser observado e avaliado pelo enfermeiro e não algo que a mãe descreveria a respeito de si.

\section{Outros dados presentes nos discursos maternos relativos ao Conflito no desempenho do papel de mãe}

Embora não tenha sido objeto deste estudo, considera-se importante mencionar que alguns fatores relacionados do DE Conflito no desempenho do papel de mãe estiveram presentes nas falas maternas. O que corrobora para o desenvolvimento de estudos a respeito deste fenômeno de Enfermagem na unidade neonatal de internação. Estes fatores relacionados, evidenciados nas falas, algumas vezes não se mostraram descritos exatamente como proposto pela NANDA-I(5).
Quanto ao fator relacionado cuidado domiciliar de uma criança com necessidades especiais, o que aparece nos depoimentos maternos é um cuidado relacionado a outros filhos pequenos no domicílio, assim como uma preocupação com o cuidado do RN após a alta, devido a necessidades especiais: como exemplo, o uso de sondas e oxigenoterapia $^{(22)}$.

A interrupção da vida familiar em virtude do regime de cuidado domiciliar (p. ex., tratamentos, cuidadores, falta de descanso) aparece na fala das mães como preocupação constante, sobretudo devido às mudanças no planejamento e do funcionamento da família como um todo(21-22).

A intimidação com relação a modalidades de tratamento restritivas e invasivas aparece em vários trabalhos como um aspecto bastante importante da vivência materna $^{(14-15,18,26)}$. O que denota a necessidade de elaborar estratégias para amenizar esta intimidação.

O fator relacionado separação da criança por doença crônica se apresenta no contexto dos trabalhos como uma separação entre mãe e criança devido à hospitalização desta última ${ }^{(11,15-16,26)}$.

As políticas dos centros de cuidados especializados, com suas normas e restrições, mostraram-se presentes também nos discursos maternos como um fator que tornou ainda mais difícil a situação vivenciada por estas mulheres $^{(15,18,26)}$.

Diferentemente do esperado, o fator relacionado $\mathrm{mu}$ dança na situação matrimonial não apareceu nos depoimentos das mães entrevistadas nestes estudos.

\section{CONCLUSÃO}

A partir da revisão da literatura, considera-se que a unidade de internação neonatal é um local bastante propício para o estudo do DE em questão, uma vez que a hospitalização de RN pode significar crise e modificações no papel que as mães esperam desempenhar junto aos filhos e família. A literatura aponta que as mães de bebês prematuros são as mais propensas a problemas devido ao nascimento antecipado e separação: situações que interferem na construção e no desempenho do papel materno.

Há nos depoimentos maternos, colhidos em estudos qualitativos sobre a experiência das mães de RN hospitalizados, dados que corroboram com as $\mathrm{CD}$ s e os fatores relacionados do DE Conflito no desempenho do papel de mãe, o que se mostrou relevante nos 15 estudos selecionados.

Os diferentes papéis exercidos por estas mulheres, como mães, esposas, donas de casa e profissionais, geram-lhes muitos conflitos, o que exacerbou o seu desgaste emocional. Suas falas descrevem conflitos sobre o dever, a necessidade 
e a emoção. As maiores dificuldades parecem ser enfrentadas por aquelas que têm outros filhos, familiares dependentes de seus cuidados e/ou aquelas que trabalham fora.

Não se tem aqui a pretensão de afirmar que as mães apresentavam o DE em questão no período em que foram entrevistadas, uma vez que seria necessária investigação individualizada e o levantamento de muitos outros dados para tal, o que deveria ter ocorrido no período em que os estudos foram desenvolvidos. Por outro lado, as falas maternas confrontadas com as CDs deste diagnóstico mostram a necessidade de se olhar com maior atenção para tal fenômeno na assistência às mães na unidade neonatal. Assim como atentar para a importância do enfermeiro capacitar-se para avaliar questões subjetivas, nomear fenômenos de Enfermagem e propor intervenções.

As falas maternas trazem evidências de que as mulheres são carentes de apoio neste processo de desenvolvimento do papel materno, em situação de hospitalização do RN, assim como no conflito que vivenciam sobre seu papel em relação ao filho e à família. Apesar de todas as discussões que abordam a humanização na assistência neonatal, ainda são incipientes as estratégias sistematizadas de apoio formal a estas mulheres e suas famílias. Na maioria das vezes, as iniciativas são pontuais e partem de alguns profissionais sensíveis à questão.

\section{REFERÊNCIAS}

1. Araújo BF, Tanaka AC, Madi JM, Zatti H. Estudo da mortalidade de recém-nascidos internados na UTI neonatal do Hospital Geral de Caxias do Sul, Rio Grande do Sul. Rev Bras Saúde Mater Infant. 2005;5(4):463-9.

2. Wereszczak J, Miles MS, Holditch-Davis D. Maternal recall of the neonatal intensive care unit. Neonatal $\mathrm{Ne}$ tw.1997;16(4):33-40.

3. Miles MS, Holditch-Davis D, Burchinal P, Nelson D. Distress and growth outcomes in mothers of medically fragile infants. Nurs Res.1999;48(3):129-40.

4. Fenwick J, Barclay L, Schmied V. Struggling to mother: a consequence of inhibitive nursing interactions in the neonatal nursery. J Perinat Neonatal Nurs. 2001;15(2):49-64.

5. North American Nursing Diagnosis Association International (NANDA-I). Diagnósticos de enfermagem da NANDA: definições e classificação 2009 -2011. Porto Alegre: Artmed; 2010.

6. Brazelton TB. O desenvolvimento do apego: uma família em formação. Porto Alegre: Artes Médicas; 1988.

7. Kimura AF. A construção da personagem mãe: considerações teóricas sobre identidade e papel materno. Rev Esc Enferm USP. 1997;31(2):339-43.

8. Bialoskurski M, Cox CL, Hayes JA. The nature of attachment in a neonatal intensive care unit. J Perinat Neonatal Nurs. 1999;13(1):66-77.

9. Belli MAJ. Assistência à mãe de recém-nascido internado na UTI neonatal: experiências, sentimentos e expectativas manifestadas pelas mães. Rev Esc Enferm USP. 1995;29(2):193-210.

10. Cunha MLC. Recém-nascidos hospitalizados: a vivência de pais e mães. Rev Gaúcha Enferm. 2000;21(n.esp):70-83.
11. Flacking R, Ewald U, Nyqvist KH, Starrin B. Trustful bonds: a key to becoming a mother and to reciprocal breastfeeding. Stories of mother of very preterm infants at a neonatal unit. Soc Sci Med. 2006;62(1):70-80.

12. Carpenito LJ. Diagnósticos de enfermagem: aplicação à prática clínica. 8a ed. Porto Alegre: Artmed; 2002.

13. Mendes KDS, Silviera RCC, Galvão CM. Revisão integrativa: método de pesquisa para a incorporação de evidências na saúde e na enfermagem. Texto Contexto Enferm. 2008; 17(4):758-64.

14. Camargo CL, La Torre MPS, Oliveira AFVR, Quirino MD. Sentimentos maternos na visita ao recém-nascido internado em unidade de terapia intensiva. Ciênc Cuidado Saúde. 2004; 3(3):267-75.

15. Centa ML, Moreira EC, Godoy MN, Pinto HR. A experiência vivida pelas famílias de crianças hospitalizadas em uma unidade de terapia intensiva neonatal. Texto Contexto Enferm. 2004;3(3):444-51.

16. Fraga ITG, Pedro ENR. Sentimentos das mães de recém-nascidos prematuros: implicações para a enfermagem. Rev Gaúcha Enferm. 2004;25(1):89-97.

17. Rocha RG, Silva ROL, Handem PC, Figueiredo NMA. Imaginário das mães de filhos internados em UTI-Neonatal no pós-parto: contribuições para a enfermagem. Esc Anna Nery Rev Enferm. 2004;8(2):211-6.

18. Heermann JA, Wilson ME, Wilhelm PA. Mothers in the NICU: outsider to partner. Pediatr Nurs. 2005;31(3):176-200.

19. Sales CA, Muriel RV, Mikuni PK, Ferreira EA, Andrade VCC, Godoy AV, Lopes NAG, Zanoni CN. Vivenciando a facticidade em dar existência a filho prematuro: compreensão dos sentimentos expressos pelas mães. Acta Sci Health Sci. 2005;27(1):19-23. 
20. Sales CA, Alves NB, Vrecchi MR, Fernandes J. Concepções das mães sobre os filhos prematuros em UTI. Rev Bras Enferm. 2006;59(1):20-4.

21. Vasconcelos MGL, Leite AM, Schochi CGS. Significados atribuídos à vivência materna como acompanhante de recém-nascido pré-termo e de baixo peso. Rev Bras Saúde Mater Infant. 2006;6(1):47-57.

22. Battikha EC, Faria MCC, Kopelman BI. As representações maternas acerca do bebê que nasce com doenças orgânicas graves. Psicol Teor Pesq. 2007;23(1):17-24.

23. Monteiro MAA, Pinheiro AKB, Souza AMA. Vivência de puérperas com filhos recém-nascidos hospitalizados. Esc Anna Nery Rev Enferm. 2007;11(2):276-82.
24. Shin $\mathrm{H}$, White-Traut $\mathrm{R}$. The conceptual structure of transition to motherhood in the neonatal intensive care unit. J Adv Nurs. 2007;58(1):90-8.

25. Lindberg B, Ohrling K. Experiences of having a prematurely born infant from the perspective of mothers in Northern Sweden. Int J Circumpolar Health. 2008;67(5):461-71.

26. Lee SN, Long A, Boore J. Taiwanese women's experiences of becoming a mother to a very-low-birth-weight preterm infant: a grounded theory study. Int J Nurs Stud. 2009;46(3):326-36.

27. Moreno RLR, Jorge MSB. O cuidar do outro na unidade de terapia intensiva neonatal: concepção fenomenológica. Ciênc Cuidado Saúde. 2005;4(3):242-9. 